\title{
SYNTHESIS STUDY OF MAGNETIC PROPERTIES AND GIANT MAGNETOIMPEDANCE IN MANGANITES NANOCRYSTALLINE $\mathbf{L}_{\mathrm{a0} .} 7 \mathrm{~B}_{\mathrm{a0.3}-\mathrm{XS}} \mathrm{XM}_{\mathrm{r} 3}$
}

\author{
da Silva, R. B.*; de Góis, M. M.; de Freitas, C. R. D.; Soares, J. M.
}

Departamento de Física, Universidade Estadual do Rio Grande do Norte - UERN, Mossoró, RN, Brasil

*Rodolfo.bezerra@hotmail.com

\begin{abstract}
Recent studies reported in the literature have shown that nanocrystalline manganites may have the effect of giant magnetoimpedance (GMI). The GMI effect was observed in the region reaching the radiofrequency values of up to $70 \%$ at room temperature in less than $1 \mathrm{kOe}$ magnetic field. Due to these characteristics, manganite may be applied to the development of magnetic sensors. In this work, we present a study of magnetic materials with nanometric manganites $\mathrm{La}_{0.7} \mathrm{Ba}_{0.3-\mathrm{x}} \mathrm{Sr}_{x} \mathrm{MnO}_{3}(0 \leq \mathrm{x} \leq 0.3)$ varying the concentration of $\mathrm{Ba}$ and $\mathrm{Sr}$. In the chemical synthesis process, we use the technique of reaction by ion coordination, setting the corresponding stoichiometric proportions of each compound. The samples were compacted in tablet form and synthesized to $1150{ }^{\circ} \mathrm{C}$ for $10 \mathrm{~h}$ in an atmosphere of $\mathrm{O}_{2}$. Analysis of X-ray diffraction (XRD) showed the formation of a polycrystalline phase with pure orthorhombic perovskite structure and space group Pbnm: cab. Through Rietveld refinements of X-ray powder diffraction patterns of samples, we obtained average particle diameters around $100 \mathrm{~nm}$. Magnetic measurements as a function of temperature with Zero-Field-Cooling (ZFC) and Field-Cooling (FC) were made using a vibrating sample magnetometer. Magnetic hysteresis cycles at room temperature showed that the samples have: low coercivity and saturation magnetization to the maximum around $73 \mathrm{emu} / \mathrm{g}$ for Sample $\mathrm{La}_{0.7} \mathrm{Ba}_{0.3} \mathrm{MnO}_{3}$. Scanning electronic microscopy images (SEM) of the $\mathrm{La}_{0.7} \mathrm{Ba}_{0.3} \mathrm{MnO}_{3}$ pellet detailing the size and shape of the particles is influenced by different pressures applied on the material in the compaction. Electrical impedance measurements as a function of frequency and magnetic field will also be presented.
\end{abstract}

